\title{
Fast and robust global decoupling with coupling angle modulation
}

\author{
Y. Luo, ${ }^{*}$ P. Cameron, A. Della Penna, Jr., J. Laster, A. Marusic, F. Pilat, T. Roser, and D. Trbojevic \\ Brookhaven National Laboratory, Upton, New York 11973, USA
}

(Received 22 February 2005; published 14 July 2005)

\begin{abstract}
We describe a fast and robust global decoupling scheme, coupling angle modulation. This novel technique introduces an extra rotating coupling coefficient into the coupled optics to determine the global decoupling strengths. The eigentune split is used as the observable during the modulation. The two eigentunes are tracked with a high-resolution phase locked loop tune measurement system. In the article, the principle of coupling angle modulation is presented, followed by its application to the Relativistic Heavy Ion Collider (RHIC). Coupling angle modulation coupling correction has been used for the global coupling correction on the nonstop RHIC ramp.
\end{abstract}

DOI: 10.1103/PhysRevSTAB.8.074002

PACS numbers: 29.20.Dh, 29.27.Bd

\section{INTRODUCTION}

A fast and robust global decoupling scheme, coupling angle modulation, is found and reported in this article. It modulates two orthogonal skew quadrupole families out of phase to introduce an extra rotating coupling coefficient into the coupled optics. The eigentune split is used as the global coupling observable [1,2]. The two eigentunes are tracked with a high-resolution phase locked loop (PLL) tune measurement system [3,4]. The global coupling correction strengths are directly determined from the modulating skew quadrupole strengths at the minimum tune split, multiplied by a factor $k . k$ is given by the minimum and maximum tune splits, together with that without the modulation.

Coupling angle modulation is a fast and robust decoupling scheme, compared to other coupling measurement or correction schemes [5-8]. According to the beam experiments in the Relativistic Heavy Ion Collider (RHIC), the modulation frequency for RHIC is $0.5 \mathrm{~Hz}$ at injection and store, $0.2 \mathrm{~Hz}$ on the ramp. Since the maximum and minimum tune splits can be determined in one coupling angle modulation period, the decoupling strengths can be found in seconds. This advantage makes this scheme viable for the global decoupling on the nonstop energy ramp of superconducting accelerators like RHIC [8,9]. It has been used to the global decoupling on the RHIC ramp in the feed-forward mode.

\section{COUPLING ANGLE MODULATION}

\section{A. Tune split}

From the linear difference coupling's Hamiltonian perturbation theory [10-13], the two eigentunes $Q_{1,2}$ under the coupled situation are

$$
Q_{1}=Q_{x, 0}-\frac{\Delta}{2}+\frac{1}{2} \sqrt{\Delta^{2}+\left|C^{-}\right|^{2}},
$$

*Electronic address: yluo@bnl.gov

$$
Q_{2}=Q_{y, 0}+\frac{\Delta}{2}-\frac{1}{2} \sqrt{\Delta^{2}+\left|C^{-}\right|^{2}},
$$

where $Q_{x, 0}, Q_{y, 0}$ are the uncoupled tunes when all coupling sources are removed. $\Delta$ is the uncoupled fractional tune split,

$$
\Delta=Q_{x, 0}-Q_{y, 0}-p,
$$

$p$ is the integer tune split. $C^{-}$is the coupling coefficient, which normally is a complex number. It is defined as

$$
C^{-}=\left|C^{-}\right| e^{i \chi}=\frac{1}{2 \pi} \oint \sqrt{\beta_{x} \beta_{y}} k_{s} e^{i\left[\Phi_{x}-\Phi_{y}-\Delta(2 \pi s / L)\right]} d l .
$$

$\left|C^{-}\right|$is the coupling coefficient amplitude, $\chi$ is the angle of the coupling coefficient. $\beta_{x}$ and $\beta_{y}$ are the uncoupled betatron amplitude functions, $\Phi_{x}$ and $\Phi_{y}$ are the unperturbed betatron phase advances, $k_{s}$ is the skew quadrupole strength, $L$ is the ring circumference, and $s$ is the distance between the skew quadruple and the reference to calculate the coupling coefficient.

From Eqs. (1) and (2) the fractional eigentune split $\Delta Q$ is given by

$$
|\Delta Q|=\left|Q_{1}-Q_{2}-p\right|=\sqrt{\Delta^{2}+\left|C^{-}\right|^{2}} .
$$

The tune split $\Delta Q$ can be measured experimentally.

\section{B. Rotating coupling coefficient}

Coupling angle modulation modulates two orthogonal skew quadrupole families out of phase to produce a rotating coupling coefficient into the coupled optics. The rotating coupling coefficient is

$$
C_{\mathrm{rot}}^{-}=\left|C_{\mathrm{rot}, \mathrm{amp}}^{-}\right| \cdot e^{i 2 \pi f t},
$$

where $f$ is the coupling angle modulation frequency, $\left|C_{\text {rot, amp }}^{-}\right|$is the rotating coupling amplitude.

Figure 1 shows the schematic plot of the coupling angle modulation. The horizontal and vertical axes are the real 


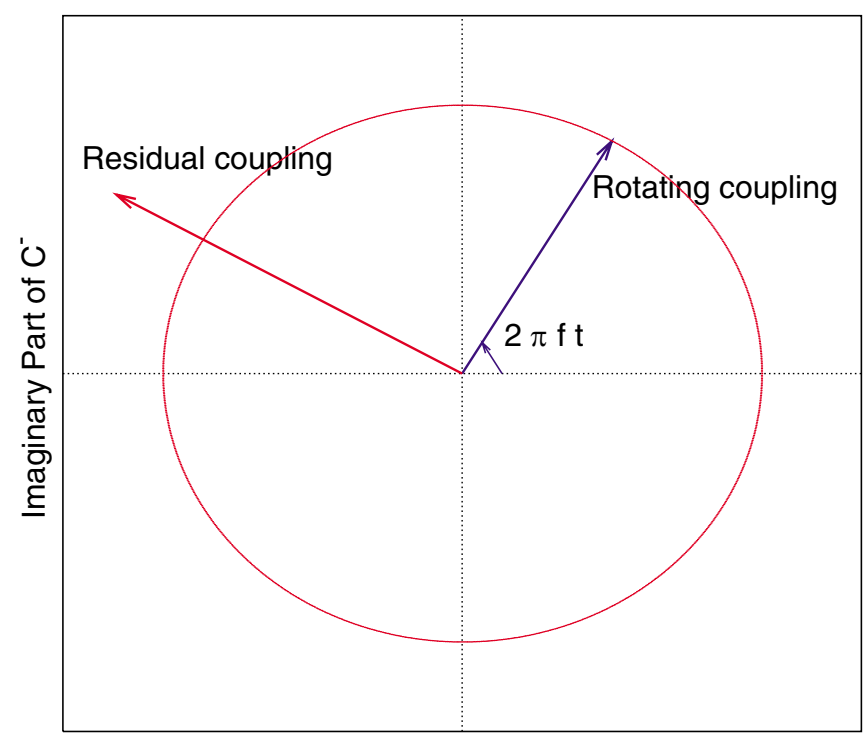

Real Part of $\mathrm{C}^{-}$

FIG. 1. (Color) Schematic plot of coupling angle modulation.

and imaginary parts of the complex coupling coefficient, respectively. The blue line represents the rotating coupling $C_{\text {rot }}^{-}$given by Eq. (6). The red line represents the residual coupling $C_{\text {res }}^{-}$in the coupled optics.

\section{Correction strengths}

During the coupling angle modulation, the total coupling coefficient $C_{\text {tot }}^{-}$is

$$
C_{\text {tot }}^{-}=C_{\text {res }}^{-}+C_{\text {rot }}^{-} \text {. }
$$

The residual coupling $C_{\text {res }}^{-}$is

$$
C_{\text {res }}^{-}=\left|C_{\text {res,amp }}^{-}\right| e^{i \phi_{\text {res }}} \text {. }
$$

According to Eq. (5), the tune split square during the modulation is

$$
\begin{aligned}
|\Delta Q|^{2}= & \Delta^{2}+\left|C_{\text {res,amp }}^{-}\right|^{2}+\left|C_{\text {rot,amp }}^{-}\right|^{2} \\
& +2\left|C_{\text {res,amp }}^{-}\right|\left|C_{\text {rot,amp }}^{-}\right| \cos \left(2 \pi f t-\phi_{\text {res }}\right) .
\end{aligned}
$$

Assuming the rotating coupling's amplitude $\left|C_{\text {rot,amp }}^{-}\right|$is constant during the coupling angle modulation, we define

$$
\left|C_{\text {res,amp }}^{-}\right|=k\left|C_{\text {rot,amp }}^{-}\right| \text {. }
$$

$k$ is a non-negative number.

From Eq. (9) and Fig. 1, we obtain the maximum tune split when the rotating coupling coefficient takes the same direction as the residual coupling coefficient, and the minimum tune split when the rotating coupling coefficient has the opposite direction to the residual coupling coefficient.

Therefore, according to Eqs. (9) and (10), the maximum and minimum tune split squares are

$$
\Delta Q_{\min }^{2}=\Delta^{2}+(k-1)^{2} \cdot\left|C_{\text {rot, amp }}^{-}\right|^{2},
$$

$$
\Delta Q_{\max }^{2}=\Delta^{2}+(k+1)^{2} \cdot\left|C_{\text {rot,amp }}^{-}\right|^{2} .
$$

Together with the tune split $\Delta Q_{0}$ without modulation,

$$
\Delta Q_{0}^{2}=\Delta^{2}+k^{2} \cdot\left|C_{\text {rotamp }}^{-}\right|^{2},
$$

the factor $k$ can be determined,

$$
k=\left[4\left(\frac{\Delta Q_{\max }^{2}-\Delta Q_{0}^{2}}{\Delta Q_{\max }^{2}-\Delta Q_{\min }^{2}}-\frac{1}{2}\right)\right]^{-1} .
$$

The factor $k$ has a significant role in determining of the global coupling correction strengths. The global coupling correction strengths are given by the modulation skew quadrupole strengths at the minimum tune split multiplied by the factor $k$.

\section{APPLICATION TO THE RHIC RINGS}

We modulate two orthogonal families out of phase to produce the rotating coupling coefficient. Each RHIC ring has three correction skew quadrupole families, F1, F2, and F3. Based on the optics model, we combine skew quadrupole families F1 and F3 to generate a new family (F1F3) that is orthogonal to the family F2. To get a $90^{\circ}$ modulation phase difference, family F2 is modulated with cos function and families F1 and F3 sin function.

\section{A. Correction at injection and store}

Coupling angle modulation correction was first tested at RHIC injection and store. Here we gave an example in the RHIC Blue ring store.

Figure 2 shows the eigentunes during the coupling angle modulation correction. The modulation amplitude for the skew quadrupole families are $\left|\left(k_{s} d l\right)_{1,3}\right|_{\text {mod,amp }}=5.77 \times$ $10^{-5} \mathrm{~m}^{-1},\left|\left(k_{s} d l\right)_{2}\right|_{\text {mod,amp }}=1.0 \times 10^{-4} \mathrm{~m}^{-1}$. The modulation frequency was $0.5 \mathrm{~Hz}$.

Figure 3 shows the modulating skew quadrupole power supply currents and the tune split square $|\Delta Q|^{2}$. The minimum and maximum $|\Delta Q|^{2}$ were determined between the two vertical grid lines which start at 5 th second and end at 7 th second. We obtained the factor $k=0.98$. The increment coupling correction strengths are $\Delta\left(k_{s} d l\right)_{1,3}=$ $4.94 \times 10^{-5} \mathrm{~m}^{-1}, \Delta\left(k_{s} d l\right)_{2}=-5.09 \times 10^{-5} \mathrm{~m}^{-1}$.

After applying the correction strengths, the eigentunes were pushed closer, shown in Fig. 2. We scanned the design tunes to measure the minimum tune split $\Delta Q_{\min }$. Before the coupling correction, $\Delta Q_{\min }=0.0078$. After coupling correction, $\Delta Q_{\min }=0.0006$. The residual coupling was considerably corrected.

\section{B. Correction on the ramp}

To decouple on the nonstop ramp is important for the RHIC performance, especially for the RHIC polarized proton (pp) run. The coupling angle modulation has been used for the RHIC global decoupling on the ramp in the feed-forward mode. 


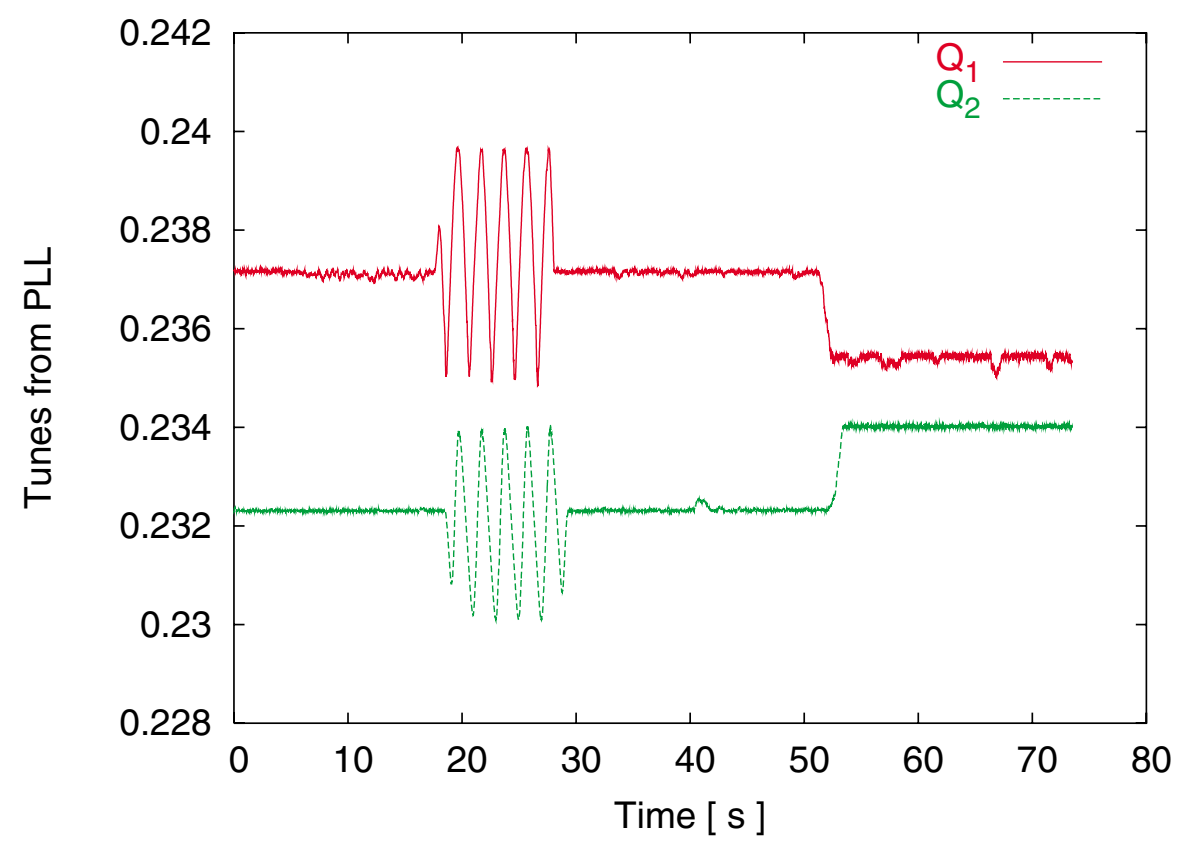

FIG. 2. (Color) The tunes during the coupling angle modulation correction in the RHIC Blue ring store.

Figure 4 shows the Yellow beam tunes in the first ramp. Three coupling angle modulations were released in the first 140 seconds' ramp. The modulation strengths were $\left|\left(k_{s} d l\right)_{1,3}\right|_{\text {mod, amp }}=8.66 \times 10^{-5} \mathrm{~m}^{-1}, \quad\left|\left(k_{s} d l\right)_{2}\right|_{\text {mod,amp }}=$ $1.5 \times 10^{-4} \mathrm{~m}^{-1}$. The modulation frequency was $0.2 \mathrm{~Hz}$. Each modulation took 15 seconds, or three modulation periods.

Figure 5 shows the tunes in the second ramp. The global coupling correction strengths from the modulations in the first ramp were applied. There was no design tune change between these two ramps. From Fig. 5, the tune split got much smaller for the second ramp. Therefore, the global coupling was reduced.

\section{DISCUSSION}

Coupling angle modulation is a fast and robust global coupling correction method. The decoupling direction and decoupling depth $k$ can be determined in one modulation period. Therefore, the correction strengths are determined

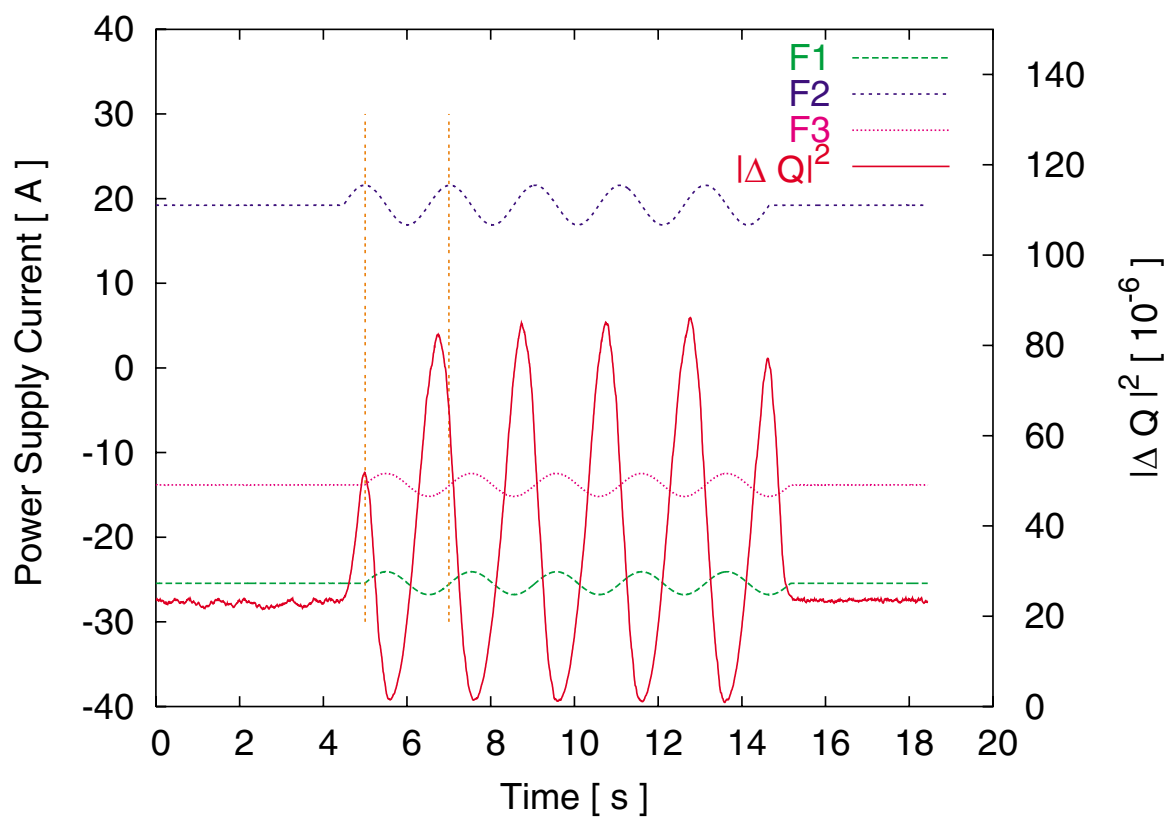

FIG. 3. (Color) The coupling correction strength searching. 


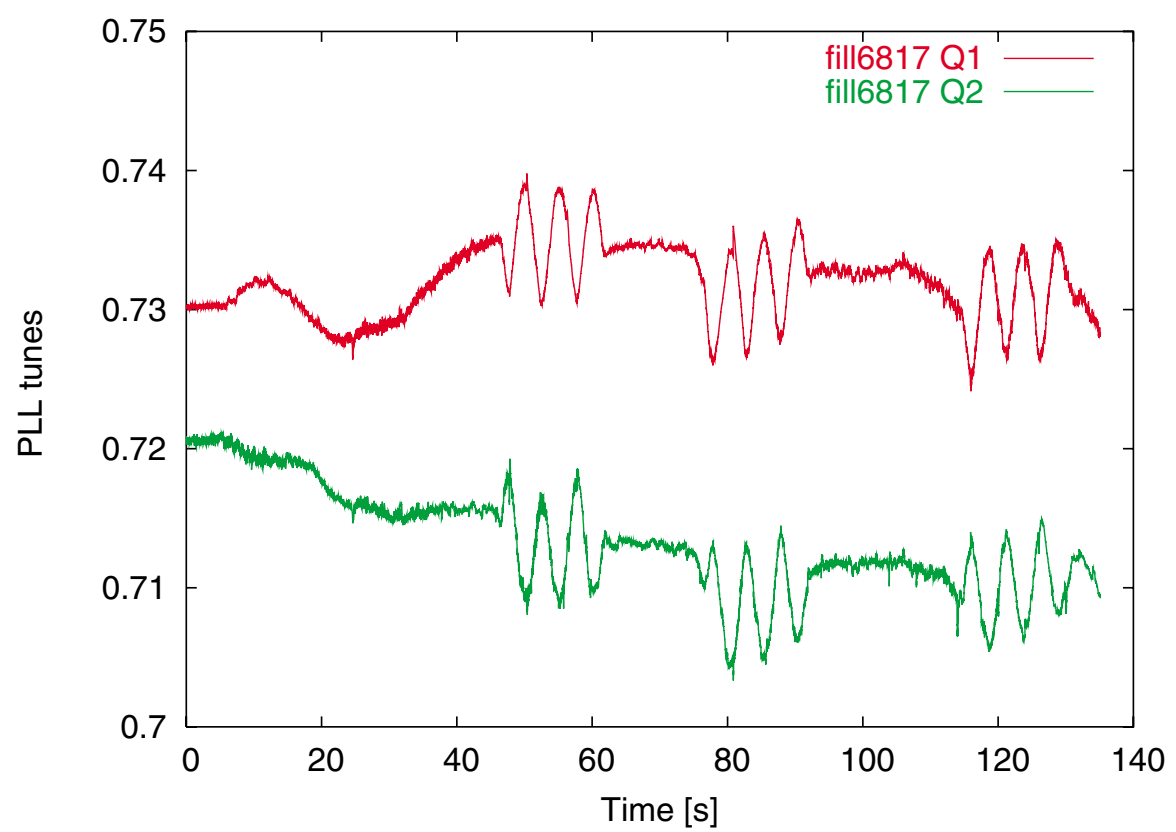

FIG. 4. (Color) The Yellow ring tunes in the first ramp with coupling angle modulations.

in seconds. This advantage makes this scheme viable for the global decoupling on the nonstop energy ramp of superconducting accelerators like RHIC.

The key point for coupling angle modulation is to produce the rotating coupling coefficient. Therefore, strict outof-phase modulations of two orthogonal skew quadrupole families is required. It is proved from simulation and beam experiment that exact orthogonal families and exact same modulation amplitudes are not strictly required. Under these nonperfect situations, the rotating coupling coefficient traces out an ellipse in Fig. 1, instead of a circle. However, Eq. (10) still holds.

The coupling correction strengths are directly linked to the modulation skew quadrupole strengths at the minimum tune split. The correction depth $k$ is decided by the maximum and minimum tune splits during the modulation. The spikes in the tune measurement can be filtered out with fitting.

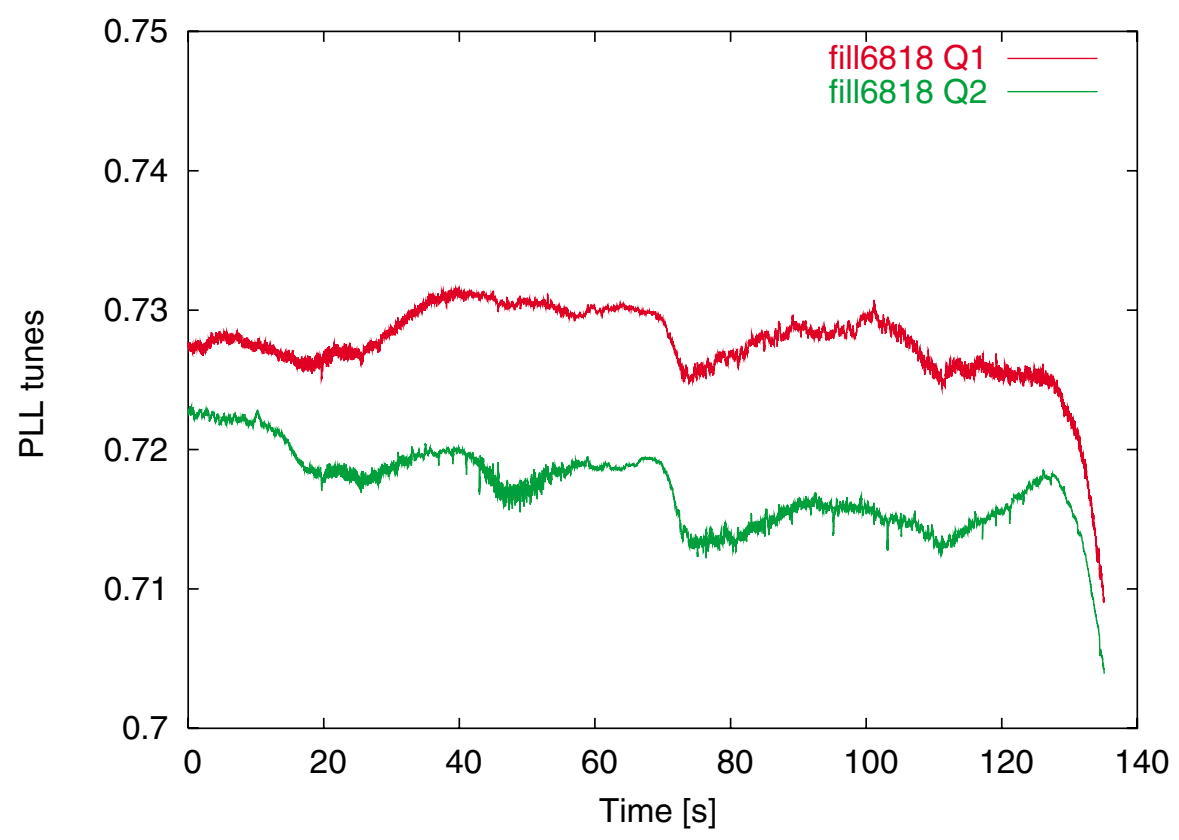

FIG. 5. (Color) The Yellow ring tunes in the second ramp with coupling corrections. 
Factor $k$ plays an important role in the determining of coupling correction depth. From Eq. (14), when $\left(\Delta Q_{\max }^{2}-\right.$ $\left.\Delta Q_{0}^{2}\right) /\left(\Delta Q_{\max }^{2}-\Delta Q_{\min }^{2}\right)$ is close to $\frac{1}{2}$, it is hard to precisely determine $k$. Difficulty in determining $k$ also happens on the ramp when the tunes and skew quadrupole strength settings change fast during the modulation.

A PLL tune measurement system is used for the tune tracking in the coupling angle modulation. PLL fails in tracking both eigentunes under some situations, especially when the two tunes are very close to each other. PLL tune losing track poses a challenge to the coupling angle modulation's application on the ramp.

Coupling angle modulation uses the eigentune split as the coupling observable [1,2]. The eigentune split is insensitive when the working point is close to the linear coupling resonance line. And to detect the residual coupling, extra coupling sources are introduced. Therefore, coupling angle modulation is not suitable for the continuous coupling measurement and correction in the feedback mode $[1,14]$.

\section{CONCLUSION}

A fast and robust global decoupling scheme, coupling angle modulation, is reported. Two orthogonal skew quadrupole families are modulated, so introducing an extra rotating coupling into the coupled optics to determine the coupling correction strengths. The global coupling correction strengths are directly obtained from the modulating skew quadrupole strengths at the minimum tune split, multiplied by a factor $k . k$ is determined by the minimum and maximum tune splits during the modulation and that without modulation. This scheme was tested in the RHIC at injection, store, and on the ramp. It has been used for the global decoupling on the RHIC ramp.

\section{ACKNOWLEDGMENTS}

The authors thank S. Peggs and V. Litvinenko for the stimulating discussions, as well as the RHIC AP group colleagues and the RHIC operation staffs for their supports during the beam experiments. And thanks goes to A. Woodhead for her help in preparing this manuscript. This work is supported by U.S. DOE under Contract No. DEAC02-98CH10886.

[1] Y. Luo, P. Cameron, S. Peggs, and D. Trobjevic, BNL CAD/AP Note 174, 2004 (unpublished).

[2] Y. Luo, BNL C-AD/AP Note 187, 2005 (unpublished).

[3] P. Cameron et al., in Proceedings of the European Particle Accelerator Conference, Lucerne, Switzerland, 2004 (EPS-AG, Lucerne, 2004), p. 524.

[4] P. Cameron et al., in Proceedings of the European Particle Accelerator Conference, Lucerne, Switzerland, 2004 (Ref. [3]), p. 1294.

[5] J. Y. Liu et al., Phys. Rev. E 49, 2347 (1994).

[6] P. F. Tavares et al., Phys. Rev. ST Accel. Beams 1, 054001 (1998).

[7] W. Fischer, Phys. Rev. ST Accel. Beams 6, 062801 (2003).

[8] Y. Luo et al., Phys. Rev. ST Accel. Beams 8, 014001 (2005).

[9] Y. Luo et al., BNL C-AD/AP Note 162, 2004 (unpublished).

[10] S. Scoch, CERN Report No. 57-20, 1957 (unpublished).

[11] G. Guignard, CERN Report No. 76-06, 1976 (unpublished).

[12] G. Guignard, Phys. Rev. E 51, 6104 (1995).

[13] H. Wiedemann, Particle Accelerator Physics II, Nonlinear and Higher-Order Beam Dynamics (Springer-Verlag, Berlin, 1995).

[14] R. Jones, P. Cameron, and Y. Luo, BNL C-AD/AP Note 204, 2005 (unpublished). 